\title{
Genetix
}

Take the lead

\section{Stem and iPS cell selection: quantitation of surface marker (SSEA1) and intracellular GFP}

\author{
Consistent and reliable propagation of undifferentiated stem cells is of particular interest in cell \\ line development and drug screening. The Genetix ClonePix FL technology, already established for \\ the rapid one-step identification and isolation of hybridomas and cell lines for biopharmaceutical \\ production, can be used to isolate and collect stem cells expressing specific cell surface and \\ intracellular proteins.
}

Recent reports of the reprogramming of fibroblasts into induced pluripotent stem (iPS) cells open up the possibility of targeted selection of cells with specific characteristics for therapeutic applications ${ }^{1-3}$. The ClonePix FL system lends itself to these specific stem cell challenges. It works by imaging thousands of mammalian cells grown into discrete clonal colonies (either grown adherently or suspended in semisolid medium). Specific fluorescent probes are then used to detect and quantify expressed or secreted proteins or peptides produced by each colony. The clones that have the desired level of fluorescence for a given colony volume (for example, the highest producers of a target protein) are then automatically collected. Up to five fluorescence wavelengths can be multiplexed with brightfield images, thus allowing multiple probes to be used in a single picking run, or to measure in parallel other parameters such as cell viability (using Genetix LiveDetect reagent). By eliminating traditional screening methods and minimizing the amount of labor required, ClonePix FL shortens the process timeline.

\section{Selection of stem cells using cell surface markers}

A powerful tool in stem cell research would be a system that could select clonal populations based on the presence of cell surface markers. One example of how this could be applied might be for the maintenance of a pluripotent cell line. Another might be to generate a cell line expressing a protein of interest, such as a heterologous receptor.

To demonstrate that the ClonePix FL is able to selectively pick colonies of cells that remain pluripotent, CGR8 mouse embryonic stem ( $\mathrm{mES}$ ) cells were plated into liquid medium and semisolid medium, and then probed with a rhodamine-conjugated antibody to stage-specific embryonic antigen 1 (SSEA1). For liquid medium, cells were plated

Christopher J Mann, Edmund N C Newman, Dominick J Whitney, Nicola J Latchem, Irene Bramke \& Julian F Burke

Genetix Ltd., Queensway, New Milton, Hampshire, BH25 5NN, UK. Correspondence should be addressed to C.J.M. (chris.mann@genetix.com).

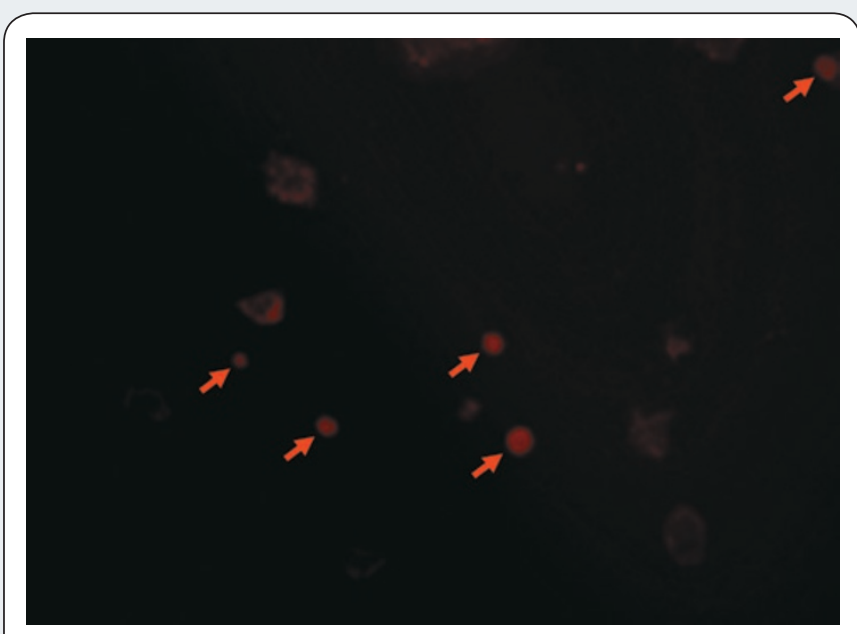

Figure 1 | CGR8 mES cells grown in CloneMatrix-based semisolid medium for $4 \mathrm{~d}$ and imaged for rhodamine-conjugated anti-SSEA1 on ClonePix FL. Pluripotent cell colonies show high expression of SSEA1 and have small, round morphology (red arrows). Differentiated clones show faint expression of SSEA1 and have larger, irregular morphology. Filter set for rhodamine: excitation, $530 \mathrm{~nm}$; emission, $590 \mathrm{~nm}$

into complete GMEM containing 10\% fetal calf serum, 1,000 U/ml leukemia inhibitory factor and $0.1 \mathrm{mM} \beta$-mercaptoethanol at a density of 500 cells per well ( $2 \mathrm{ml}$ ) of a black-walled Genetix PetriWell-6 tissue culture treated plate and colonies were grown to a diameter of 1-2 mm. Medium was changed every $48 \mathrm{~h}$. On day 9 , medium was removed and replaced with $300 \mu \mathrm{l}$ of the anti-SSEA 1 detection antibody ( $10 \mu \mathrm{g}$ per well) and the plates incubated for $5 \mathrm{~min}$ to allow binding. Cells were then overlaid (without removing the antibody) in $2 \mathrm{ml}$ of medium and incubated for $2 \mathrm{~h}$ before picking. For semisolid medium, cells were grown in Genetix CloneMatrix-based semisolid medium (IMDM containing 20\% fetal calf serum, 1,000 U/ml leukemia inhibitory factor and $0.1 \mathrm{mM} \beta$-mercaptoethanol) at a density of 1,000 cells per well ( $2 \mathrm{ml}$ ) of a black-walled Genetix PetriWell-6 


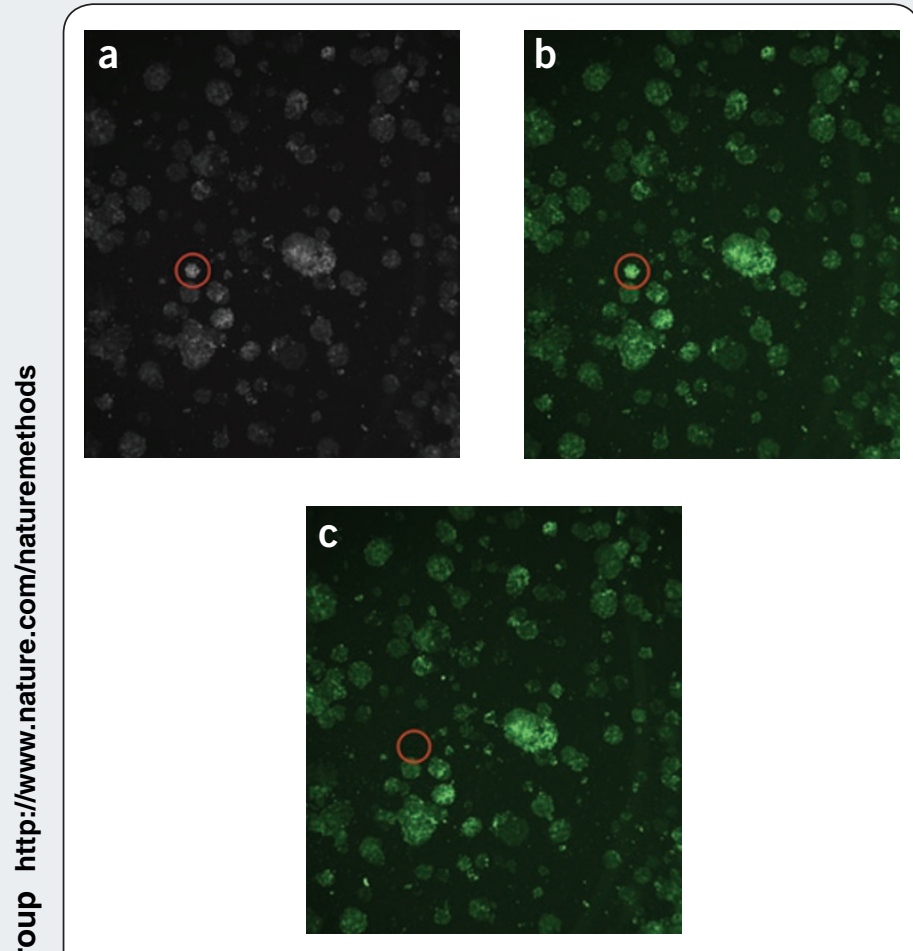

Figure $\mathbf{2}$ | Detection and picking of adherent MCF-7 clonal colonies expressing highest level of GFP. (a-c) Colonies imaged on ClonePix FL before picking in bright-field (a) and for GFP (b) and then after picking for GFP (c). Colonies were automatically collected without the use of trypsin. The selective collection of a high-expressing colony is indicated (red circle). Filter set for GFP: excitation, $470 \mathrm{~nm}$; emission, $535 \mathrm{~nm}$

non-tissue culture treated plate and grown for $3 \mathrm{~d}$. The SSEA1 detection antibody was sprayed on using a Genetix atomizer (10 $\mu \mathrm{g}$ per well) and the plates were incubated for one more day to allow detection of SSEA1 (Fig. 1) before picking.

For both adherent and suspension conditions, clones expressing the highest intensity of anti-SSEA1 were selectively collected following standard ClonePix FL picking procedures. In both cases, the picked clones showed excellent outgrowth at the 96-well stage. The picked material did not show increased differentiation after the mechanical action of picking the colonies, nor as an effect of binding of antibody to the SSEA-1 receptor. The resultant picked cells were not only clonal but also undifferentiated, and thus suitable for further propagation. Notably, the cells grown in semisolid medium showed fewer differentiated clones than those grown as adherent colonies.

\section{Selection of cells by detection of intracellular protein markers}

In addition to detection of cell surface markers, proteins expressed intracellularly can also be directly quantified using the ClonePix FL if the protein of interest is tagged with a fluorescent marker. To demonstrate this principle, a population of adherent human breast

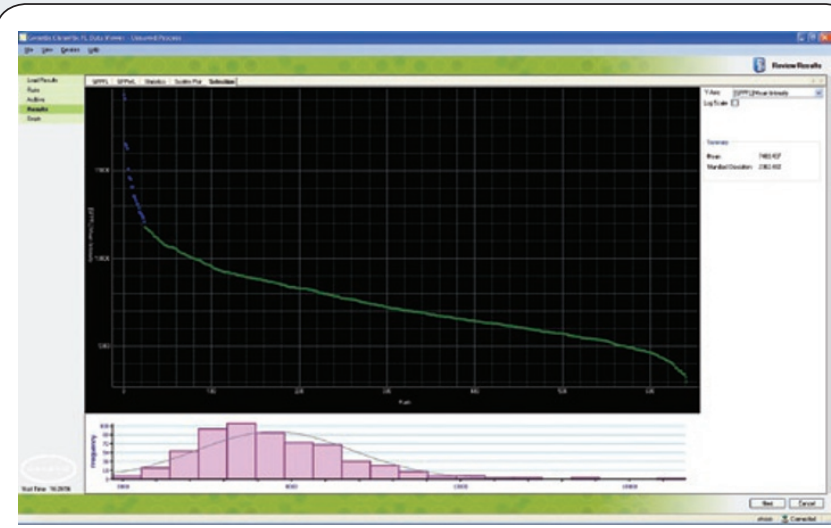

Figure $\mathbf{3}$ | Screen shot of graphical analysis generated by ClonePix FL to assist in the decision as to which clones to pick. In this example, 640 detected colonies were ranked by GFP intensity and the highest-scoring 20 were selected for picking (highlighted in blue).

cancer cells (MCF-7) expressing Aequorea coerulescens GFP were plated into black-walled Genetix PetriWell 6-well plates in MEM Earle's liquid medium with $10 \%$ fetal bovine serum. After $8 \mathrm{~d}$, the plates were imaged using ClonePix $\mathrm{FL}$, and the colonies expressing GFP most highly were selectively picked by the ClonePix FL (Fig. 2). The decision as to which colonies to collect was facilitated by graphical analysis (Fig. 3). We propose that this system could provide a powerful alternative to fluorescence-activated cell sorting (FACS) for obtaining GFP-expressing clones, such as Nanog-selectable fibroblasts ${ }^{1}$, in a single step.

\section{Conclusions}

The work presented here indicates the feasibility of using ClonePix FL technology for the selection and propagation of stem cells or iPS cells, ensuring that only pluripotent colonies are propagated further, and removing the laborious bottlenecks associated with FACS selection and stem cell propagation. The use of semisolid medium increased the number of undifferentiated colonies (presumably because the cells did not adhere to the plate surface), eliminating the need for regular medium changes. The capacity to investigate in parallel markers for pluripotency, differentiation pathways and intracellularly expressed selection markers, as well as physical morphology, provides a powerful tool for both cancer and stem cell research.

1. Maherali, N. et al. Directly reprogrammed fibroblasts show global epigenetic remodeling and widespread tissue contribution. Cell Stem Cell 1, 55-70 (2007).

2. Wernig, M. et al. In vitro reprogramming of fibroblasts into a pluripotent ES-celllike state. Nature; published online 6 June 2007 (doi:10.1038/nature05944).

3. Okita, K., Ichisaka, T. \& Yamanaka, S. Generation of germline-competent induced pluripotent stem cells. Nature; published online 6 June 2007 (doi:10.1038/nature05934).

This article was submitted to Nature Methods by a commercial organization and has not been peer reviewed. Nature Methods takes no responsibility for the accuracy or otherwise of the information provided. 